\title{
Human Ability to Learn According to the Feldenkrais Method: From Writings to Practice
}

\author{
Gil Iris \\ Correspondence: Gil Iris, Shaanan College, Ohalo College, Israel. \\ Received: November 24, 2020 \\ Accepted: January 20, $2021 \quad$ Online Published: January 23, 2021 \\ doi:10.11114/jets.v9i2.5138 \\ URL: https://doi.org/10.11114/jets.v9i2.5138
}

\begin{abstract}
The Feldenkrais method seeks to improve individual ability, and human functioning in general, through movement. According to Moshé Feldenkrais, refining movement by enhancing the nervous system's functioning also improves our ability to learn. The method has already been shown to improve mood, quality of life, and various physical aspects. The present study seeks to examined students' theoretical learning capabilities during a Feldenkrais lesson, during a period of a few hours after the lesson, and the correlation between these two time periods.

The main research question was: Does doing Feldenkrais exercises improve the ability to learn in 120 students at Technion-Israel Institute of Technology. The findings show that the students assimilated the theoretical content conveyed during the lessons. The study also found that most of the students experienced improved ability for theoretical learning after the lessons. A strong, positive correlation was found between the students' high ability for theoretical learning during the lesson, and improvement in the learning ability of the same students, experienced after the lesson. The study's conclusion is that a variety of internal, physiological and mental/emotional processes occur when doing Feldenkrais exercises, which alleviate pain, create new neural pathways between the brain and the body, and also "unblock" stressful neural pathways, and which significantly improved the participants' concentration, attention, and theoretical learning abilities, both during and after the lessons.
\end{abstract}

Keywords: Feldenkrais method, human learning process, improving theoretical learning, inner guide

\section{Introduction}

\subsection{The Rationale of the Feldenkrais Method}

Dr. Moshé Feldenkrais developed a unique method that consists of treatment and exercises, and a human learning theory (Gil, 2013). According to Feldenkrais, we all have an "inner guide", a kind of inner sense that guides us, when we are still babies, in discovering how to move, roll over, crawl, and so forth, without explicit instruction or guidance from a teacher, or by imitation. We do so through trial and error. For instance, we experiment with different ways to raise our body from a prone position onto our hands and knees, trying out countless movements, and strengthening various muscles, until we find the way most suited to us. However, for a variety of reasons, in the socialization process typifying our development during childhood, we "bury" the inner guide that initially steered us (Alon, 1994; Feldenkrais, 1967, 1985).

By using seemingly simple processes of movement, participants are instructed to pay attention to details that usually go unnoticed: Do we always move the same way? What about our breathing? Which parts of our body are being strained? What are we feeling in our lower back and other areas? What is the direction of our head during a movement? Does the location of a particular limb, or its participation or non-participation in the exercise, change our sensations? At the conclusion of the exercise, participants are instructed to lie on their back and pay attention to the changes taking place within them, and to distinguish between different nuances (Alon, 1994; Feldenkrais, 1967).

Practicing this method enables exploration of our primary learning model, which consists of experimenting multiple times with the same movement; learning from mistakes; guidance in discovering new things in a movement; distinguishing subtle differences when doing the exercise in different ways; experimenting with variations of particular aspects of the same movement; guidance in finding multiple movement solutions for the same aspect (e.g., getting up sideways with the head leading the movement, with a different part of the body leading the movement, using hands, not using hands); experimenting with diverse variations, and, after experimentation, gaining an understanding of what is beneficial for us (e.g., lying on the back, moving upraised arms from side to side - moving the head in the same direction, then in the opposite direction); gentle exercises; a relaxed atmosphere during the lesson; and alleviating muscular strain by lying on 
the back and neutralizing the force of gravity. By means of these exercises, we create balance dynamically and multidimensionally between the body's various systems since all parts of the body are involved in the movements, including the proprioceptive (sensory), vestibular (balance), and visual (vision) systems (Alon, 1995; Feldenkrais, 1967; Teixeira-Machado et al., 2015).

Feldenkrais exercises stimulate the body's awareness through exercises directed toward harmonious, easy-to-perform functional motor movements (Teixeira-Machado et al., 2015). They also stimulate our inherent wisdom concerning how to use our body in a way that is beneficial to us, in motion and at rest, and enable us to awaken that inner guide that has been buried deep within us for many years. This guide knows how to choose the best movement for us from a range of movement possibilities, and guides us toward better functioning (Alon, 1994). The improvement that occurs is not solely due to corrected movement, but also to reintegration of human learning methods. The lessons constitute an incubator for natural refinement, for finding independent inner solutions resembling our primary developmental discovery in infancy (Alon, 1994; Feldenkrais, 1967; Gil, 2013).

\subsection{Review of the Research}

The Feldenkrais method gained widespread recognition as a highly effective method to improve awareness, health, and functioning (Alon, 1994; Gil, 2013; Hillier et al., 2015). Research on the method's efficacy in physical, emotional, and mental aspects, has found it to be highly effective. However, attempts to find evidence regarding Dr. Feldenkrais's human learning theory, as it appears in his writings, research articles, and reviews, yielded no results.

It seems that it is not only in the general population that there is a lack of awareness regarding the learning process that occurs while doing the exercises; it is unclear whether there is awareness among Feldenkrais teachers regarding this profound and intriguing learning process. For example, a study conducted by Buchanan, Nelsen, and Geletta (2014) investigated why Feldenkrais teachers chose this particular method as a profession, found that the primary reason was to treat pain. The aspect of improving and refining ability to learn was not even mentioned.

Much has been written about the method's emotional aspect (Gil, 2013). A study conducted by Teixeira-Machado et al. (2015), for example, examined the effects of practicing the Feldenkrais method on quality of life and wellbeing in fifteen adult patients (aged 50-70) with Parkinson's disease. The researchers hypothesized that doing exercises entailing gentle, enjoyable movements would have a positive effect on the patients' emotional wellbeing and everyday movement. Indeed, their results showed significant improvement in the participants' quality of life scores $(\mathrm{p}=.004)$, and significant reduction in depression levels compared with a control group, which received theoretical instruction during this period on how to cope with Parkinson's (medication, how to avoid falling, etc.). Although researchers acknowledge the method's significant general benefits, and despite proof of its efficacy among Parkinson's patients, there is no inquiry, study, exposure, or acknowledgement of the human learning process as it is presented in the writings of Dr. Feldenkrais.

This learning process does not feature in the latest studies on the method's efficacy for mental functioning either. In the past few years, medical professionals, including surgeons, refer to the Feldenkrais method as one that stimulates and regenerates the brain (McIvor, 2015; Morgan, 2017).

The current approach to coping with chronic pain combines conventional medicine and alternative therapies. This approach also includes the Feldenkrais method as a physical treatment method that provides long-term muscle relaxation (Chen, 2017). Morgan (2017) studied the method, and found that participating in twelve Feldenkrais lessons reduced chronic pain. According to the combined approach, the Feldenkrais method heightens awareness of skeletal muscle structures (relationships), and can result in correction of these structures.

However, this important insight, namely that various physical improvements stemming from participating in Feldenkrais lessons are probably the result of an independent inner learning process, can be seen in the review conducted by Hillier and Worley (2015). They reviewed twenty studies about the Feldenkrais method conducted over the past twenty years, from 1991 (Kegerreis \& Brown) to 2014 (Nambi, Trivedi, Momin, Patel, \& Pancholi). The researchers sought to examine the Feldenkrais method's advantages by reviewing studies on its efficacy in various aspects and populations using a variety of intervention programs. Some of the studies engaged with healthy populations (Bitter, Hillier, \& Civetta, 2011; Ruth \& Kegerreis, 1992; Stephens, Davidson, DeRosa, Kriz, \& Saltzman, 2006), others with aspects of elderly health (Hillier, Porter, Jackson, \& Petkov, 2010; Ullmann, Williams, Hussey, Durstine, \& McClenaghan, 2010; Vrantsidis et al., 2009), some engaged with multiple sclerosis patients (Chinn, Trujilo, Kegerreis, \& Worrel, 1994; Smith, Kolt, \& McConville, 2001; Stephens, DuShuttle, Hatcher, Shmunes, \& Slaninka, 2001), and a few with elderly institutional residents (Nambi et al., 2014), while some engaged with eating disorders (Laumer, Bauer, Fichter, \& Helmut, 1997), and myocardial infarction patients (Löwe et al., 2002).

All the reviewed studies investigated whether participating in Feldenkrais lessons can contribute to various physical proficiencies, e.g., lengthening the hamstring muscles, improving mobility and balance. In summary, these studies found 
that doing Feldenkrais exercises can improve balance and prevent falls, both in elderly populations already experiencing falls, and as prevention in adult populations not yet experiencing falls. The researchers also found evidence that Feldenkrais exercises can improve physical mobility and stimulate awareness, which helps to reduce strain and pain. The results on lengthening the hamstring muscles were inconsistent.

Hillier and Worley (2015) concluded that these benefits stem from the fact that the Feldenkrais method promotes awareness, relaxation, and more efficient mobility. According to them, the method provides general benefits, rather than treating a specific disease or difficulty, and these benefits are probably based on a process of personal learning and selfimprovement. It is important to note that the researchers reached this important conclusion without referring to the learning process itself.

The present review of the research indicates that researchers understand that a learning process of some kind occurs in people participating in Feldenkrais lessons. However, it also shows that Feldenkrais students and teachers are oriented to beneficial physical and emotional outcomes, rather than the learning that occurs during, and as a consequence of, these lessons. To examine this important aspect, we should first explore how Dr. Feldenkrais understood human ability to learn, and the learning that can occur in students and practitioners of his method.

\subsection{The Human Learning Process According to Dr. Feldenkrais's Writings}

In humans, the ability to perform visible practical functions - walking, movement, posture - is acquired over the course of several years, while in animals, most of these abilities are already present at birth, or acquired shortly afterward. Where does this slowness in human ability to learn stem from?

\subsubsection{The Human Nervous System}

One possible reason is the size of the human brain. Unlike animals, there is a huge difference between the weight of a newborn's brain (300 gram) and that of an adult (about 1,360 gram). From an observation of animals, it may be concluded that the closer the weight of a particular animal's brain at birth to that of a mature animal of the same species, the greater the similarity in its ability to function at birth and as a mature animal.

The immense increase in the weight of a baby's head during the first year, and in general, stems from the multiplication of neural pathways. In its initial state, the nervous system and all its structures - brain, neurons, and synaptic connections between neurons - are ready for action, but most of the pathways between them are not yet formed. In other words, a huge part of our nervous system is likely to develop in accordance with environmental demands: speech, movement, mimicry, and others. Thus, the environment plays a significant role in our learning. In the years after birth, the nervous system grows, develops, and can perform increasingly complex actions.

When learning new reactions, the human brain enables the individual to experiment with different options (e.g., sitting up from lying down), and after multiple experiments, the brain chooses one reaction, or a small number of reactions, that it will continue using. In other words, the human brain transforms learning processes - acquisition of new reactions - into regular, natural actions (Feldenkrais, 1966, 1993).

The human brain has three systems: (1) The rhinic system supplies the individual internal requirements of every living organism, e.g., satisfying internal needs like hunger and thirst. When not satisfied, these needs may intensify, and, when satisfied, they are reduced or abated until the need arises again, "instincts"; (2) the limbic system is a group of mainly inherited structures that complement instincts. For instance, some newborns need to be stimulated before they start sucking. This system facilitates a degree of adapting to the environment - the beginnings of the ability to learn and understand. Some animal species have a limbic system, while others do not. For example, some bird species adapt themselves to building nests from new materials when they are moved to new surroundings, while others do not manage to adjust; and (3) the supralimbic system comprises the neural pathways originating in the cerebral cortex, which distinguish humans from animals. The basic structure of this system is inherited, but its function largely depends on individual experience. This system enables the individual to play music, write, and so on. The neural pathways in this system are longer and more complex than in the first two systems, and most of its actions are performed by means of the first two. The supralimbic system enables some actions to be controlled, e.g., shall I raise my hand, or not, and it allows the individual to learn their self (Feldenkrais, 1967, 1976; Gil, 2013).

\subsubsection{Development and Learning}

In the first stage, humans develop "naturally", we all have an "inner guide", a sense that guides us to do what we need to: walk, run, talk, etc. (Alon, 1967; Feldenkrais, 1967). In the second stage, individuals develop and refine learning that occurred in the first stage; people find unique ways to do what comes naturally, e.g., painting, movement. In the third stage, certain refinements established by an individual in the second stage, become common knowledge, an acknowledged method. For example, Leonardo da Vinci used principles of perspective, which were only fully defined in the nineteenth century (by Monge), and have been taught as a discipline at every art school ever since. 
If we wish to check, and even correct, ourselves in order to create a life that is consistent with our structure and traits (first and second stages), rather than living solely in accordance with our current accidental configuration (third stage), which has been formed virtually without our knowledge, we can do so by means of our consciousness. With consciousness, the individual can review all their fields of action, and it enables them to find modes of acting and behaving that are suited to their needs (Feldenkrais, 1967, 1976; Gil, 2013).

\subsubsection{Education}

"There are many kinds and ways of learning $[\ldots]$ There is the learning of a skill [...] it can go on for more or less lengthy periods of time, apparently aimlessly, and then a new form of action appears as if from nowhere" (Feldenkrais, 1993, p. xiii).

Education, according to Dr. Feldenkrais's theory, consists of three factors that together comprise "self-image". (1) Heritage - biological quality, form, the nervous system's capacity, skeletal structure, skin, and senses; (2) external education determines our language, perception, and some of our reactions; and (3) self-education is influenced by external education, but is more active, and determines character traits, preferences, and so on. Self-image, according to which we behave, act, and live, is formed between external and self-education (Feldenkrais, 1967, 1976; Gil, 2013).

"Learning is turning darkness, which is the absence of light, into light. Learning is creation. It is making something out of nothing. Learning grows until it dawns on you" (Feldenkrais, 1993, p. 56).

Dr. Feldenkrais's theory of human learning is comprehensive and fascinating. Since other advantages of the method physical aspects such as balance, and mental aspects such as reducing stress and improving mood - have been extensively studied, the present study engages, for the first time, with the correlation between participating in Feldenkrais lessons and ability for theoretical learning.

\subsection{Research Aim and Questions}

To examine students' theoretical learning abilities during a Feldenkrais lesson, during the hours following the lesson, and the correlation between these two time periods.

1) Does theoretical learning occur during a Feldenkrais lesson?

2) Is there improvement in theoretical learning during a period of a few hours after the lesson?

3) Is there a correlation between theoretical learning during a Feldenkrais lesson, and theoretical learning during a period of a few hours after the lesson?

With reference to Research Question 1, it should be noted that the participants were not obliged or instructed to assimilate and memorize the theoretical material taught during the lesson while doing the exercises. Also, they do not learn the theoretical material in the "usual" way (i.e., writing, recording, practicing, memorizing, taking exams).

\section{Method}

\subsection{Research Approach}

Due to the fact that the research involves professional practice, and that some of its goals are to examine, develop and promote the researcher's fields of interest, a pragmatic paradigm was found to be the most suitable. Therefore, a mixedmethods research approach was chosen since it combines qualitative and quantitative approaches and tools (Creswell, 2009).

Research Questions 1 and 2 were examined by means of a qualitative tool, namely an online questionnaire, and analyzed by means of grounded theory, i.e., category analysis. Research Question 3 was examined by means of a quantitative tool, Spearman's rank correlation test. Qualitative tools facilitate understanding of the processes the participants experienced, while the quantitative tool is designed to enrich and reinforce the findings of the qualitative tools (Creswell, 2009).

\subsection{Research Stages}

The study was conducted during winter semester 2017, and spring semester 2018. Four Feldenkrais courses were held during this period, with 30 students in each course, a total of 120 students. Six questionnaires were administered during each semester, a total of 720 questionnaires, which were administered at the end of each lesson, and returned within twenty-four hours. A total of 680 questionnaires were completed and returned. Data analysis referred to data collected after three lessons, i.e., about 360 questionnaires. 
Table 1. Content of lessons in the study

\begin{tabular}{c|l|l}
\hline Lesson \# & Subject of lesson & Content of lesson \\
\hline 1 & $\begin{array}{l}\text { The importance of lying on the } \\
\text { stomach }\end{array}$ & $\begin{array}{l}\text { Reenacting the movements of babies lying on their stomach during the } \\
\text { first few months of their life }\end{array}$ \\
\hline 2 & $\begin{array}{l}\text { The relationship between the } \\
\text { head, shoulders, and neck }\end{array}$ & $\begin{array}{l}\text { Practicing joint and separate head and shoulder movements, using the } \\
\text { neck }\end{array}$ \\
\hline 3 & $\begin{array}{l}\text { The four components of the } \\
\text { waking state }\end{array}$ & $\begin{array}{l}\text { Focusing on movement as the component that is easiest to change, and } \\
\text { which can influence the three other components }\end{array}$ \\
\hline
\end{tabular}

Lesson \#1: Lying on the stomach

According to the Feldenkrais method, the entire evolution of our movement and posture as humans originates from lying on our stomach. It enables babies to go through all their development stages - raising their head; raising their head and shoulders and leaning on their forearms; raising their head, shoulders, and back, and leaning on their hands; raising their arms and legs at the same time; raising their arms and legs at the same time, and swinging their body right and left, none of which they can do when lying on their back. Experimenting with these stages enables the nervous system to learn to send correct instructions to the back muscles, so they function in a way that allows us to assimilate the correct posture. Learning correct posture in infancy can accompany us for the rest of our life. Also, by experimenting, babies experience and practice independence, confidence, and interest.

Lesson \#2: The relationship between the head, shoulders, and neck

In most people, the head, which should be positioned above the spine, tends to angle too far forward, causing deformities, tension, pain, and even damage to the cervical vertebrae. When the head is angled too far forward, the shoulders also angle too far forward. The Feldenkrais method offers multiple processes of movement to help position the head and shoulders further back, thus considerably reducing tension in the area. In the lesson on this topic, gentle exercises were performed, combining head and shoulder movements in the same direction and in opposite directions, both when lying on the back and when sitting.

\section{Lesson \#3: The four components comprising the waking state - thought, feeling, sensation, movement}

Feldenkrais focused on movement as the component through which the three others may be improved in order to refine ourselves. He chose movement since it is more difficult to direct, change, and assimilate an unusual movement than to intervene in our thoughts, feelings, or sensations. According to Feldenkrais, refining movement serves as a catalyst for refinement of the three other components (sensation, feeling, thought).

\subsection{Participants}

The participants were 120 BA students from different faculties at Technion-Israel Institute of Technology, aged 18-28 (average age: 24).

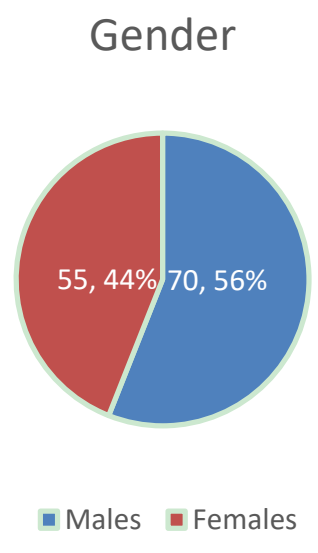

Figure 1. Distribution of Participants by Gender 


\subsection{Sampling Procedure}

A convenience sampling method was used since the participants were students in the researcher's study group. Technion students are typified by high intellectual curiosity and politeness. Most of the students attending these classes were nearing completion of their BA studies. This probably afforded them a degree of maturity and availability to participate in experiential learning, which vastly differs from the rest of their classes and lectures. This participant profile contributed to the relative ease of collecting the data for processing and analysis.

\subsection{Research Tools}

\subsubsection{Qualitative Questionnaires}

The questionnaires were specifically developed for the present study, and included questions about the participants' understanding of the theoretical content conveyed during the lesson concurrently with the practical exercises, questions about their ability to learn after the lesson, and various general questions suited to the character of the specific lesson, e.g., the sensations they experienced during the lesson. All the participants completed the same questionnaires, but each questionnaire was planned in accordance with the character and content of the lesson; thus, after each lesson, the participants responded to different questions. The aim was to spark interest and motivation to respond, and also to obscure the aim of the study in order to avoid desirability bias. Each questionnaire consisted of four to eight questions.

The reliability of the qualitative measures was the researcher herself since it is unreasonable to expect other researchers to obtain the same results from these questionnaires. There was substantial variance between the participants, and it is virtually impossible for one individual to express the same knowledge and sensations experienced in the lesson as another. All the questionnaires, their documentation, categories of analysis, and content, have been preserved.

\subsubsection{Quantitative Analysis - Spearman's Rank Correlation Test}

This test examined the correlation between theoretical learning during a Feldenkrais lesson, and theoretical learning after a Feldenkrais lesson. Each participant's responses were quantified with reference to Research Questions 1 and 2 into one overall score for theoretical learning during the lesson, and one overall score for theoretical learning after the lesson. The score ranged from 1 to 5: 1=No learning at all; 2=Hardly any learning; 3=Partial learning; 4=Some learning; and $5=$ Significant learning.

The reliability of the quantitative analysis with Cronbach's alpha was 0.77 , i.e., high reliability. The sig. (p) value in the quantitative analysis was less than 0.01 , indicating the validity of the study. In other words, the measuring tool examines the research question appropriately.

\subsection{Analysis of the Questionnaires}

Analysis of the qualitative questionnaires in the present study was based on the model proposed by Strauss and Corbin (1990), i.e., grounded theory. The analysis comprised several stages: a full reading of the data, removing non-relevant information, coding relevant information by reducing the data and organizing it according to themes, organizing the data in smaller units (categories). In some categories, sub-categories emerged (this division stemmed from the research subject and questions, and facilitated a broadening of the data by restructuring it), a rereading of the initial data, verification of the findings, and the writing stage. This form of data collection facilitated methodical examination and tracking of the study's principal ideas.

Analysis of the quantitative questionnaires was carried out using SPSS software.

\section{Findings}

\subsection{Assimilation of Theoretical Material Taught During the Lesson}

The first theme, which engaged with learning theoretical content explained during the lessons, was divided into a number of categories, each with reference to learning a particular topic.

The first category referred to processes of movement wherein the participants lay on their stomach and slowly raised various limbs. In the sub-category examining the importance of lying on the stomach and the movements performed in this position, it is evident that the participants assimilated the theoretical content, expressed in statements such as: "I learned that lying on the stomach is very important, especially as babies at the start of our life ..."; "I learned that lying on the stomach contributes to building babies' muscles that are essential for us to stand steadily and develop correctly".

Another sub-category, which also indicated good ability to learn during the lesson, engaged with the participants' ability to relate and interpret their personal experience of lying on their stomach as babies (by asking their parents), and their posture today. This is evidenced by the participants' responses: "Yes, my parents made sure to also put me on my stomach... This has important and evident influence on my motor functioning today, and on my rapid development as a baby"; "My parents did put me on my stomach when I was little, and therefore I assume my motor abilities are quite good". 
In two instances, however, the responses indicated possible difficulty in understanding the connection between lying on their stomach as babies and their present posture. "My parents say they put me on my back when I was a baby, which possibly contributed to the development of my back and neck muscles"; "I was put on my back as well. It apparently helped since I don't have back problems". Since only two responses indicated a lack of understanding, in contrast with dozens attesting to correct projection of the importance of lying on their stomach in infancy for their posture as adults, it may be concluded that the participants understood the content conveyed during the lesson.

The second category analyzed learning about the relationship between the head, shoulders, and neck. It seems that some learning occurred in most of the participants, but it was not comprehensive. On the one hand, some responses included statements such as: "In the last lesson I learned how to sit correctly in terms of the back's erectness and other parts of the body in relation to it"; "Yes, we learned how to relax the shoulders and neck, which I believe will help me later on". While on the other, they included statements like: "It was very hard for me to relax my muscles in this lesson because I was too cold in spite of the warm clothes I was wearing"; "I didn't learn".

The third category in the first theme engages with assimilation of Dr. Feldenkrais's reference to the four components comprising the waking state (Feldenkrais, 1967): thought, feeling, sensation, movement. Since all the responses in this category indicated assimilation of the importance of movement as the component that helps to bring about change in the three others, and no statements indicating no learning of the subject were identified, it may be concluded that the participants assimilated the topic: "I learned about the four components of existence according to Feldenkrais (feeling, sensation, movement, and thought). He said movement is the easiest to change (changing the others is more complicated or even impossible)"; "Yes, I learned about the thoughts, feelings, sensations, and movements comprising us, and how important it is to instill correct movement at a young age".

To sum up this theme, it appears that the participants who experienced learning new content in an unconventional way learning in motion - assimilated the theoretical content conveyed during the lessons.

\subsection{Theoretical Learning Ability During a Period of a Few hours After the Lessons}

Four interconnected and interrelated categories were defined with reference to Research Question 2. In the first category, the participants were asked a general question: "Do you feel improvement in your ability to learn after participating in the lessons?" About $70 \%$ of the participants who answered this question, responded with a definite "yes", about $20 \%$ with a definite "no", and the remaining 10\% responded "I don't know".

The second category examined how this improvement manifested in the participants who responded to the previous question with a "yes". The participants gave varied responses, which can be divided into two main sub-categories: mental/emotional and physiological. In the mental/emotional sub-category, the participants mainly emphasized improved concentration: "I think the improvement manifests in patience or concentration"; "I feel much calmer after the lesson, which leads to improved concentration, and I feel I can study for longer without taking a break".

In the physiological sub-category, it is evident that the changes mainly manifested in the participants' ability sit for longer without experiencing pain, and a sense of greater relaxation in the body: "Sitting felt more erect and correct"; "I felt like last week, that I could sit for longer, and study without my back hurting"; "Yes, at the conclusion of the lesson I feel lighter and more relaxed than when it began. These physical sensations also affect my mood during the day".

Among the answers that may be classified as "physiological", there were also two that do not attest to any significant improvement: "Learning wasn't as good as usual (does not relate it to the lesson)"; "I really try to maintain erectness throughout the day after the lesson, and that breaks my concentration a tiny bit".

In the third category, the participants were asked: "What, in your opinion, are the reasons for the improvement?" The responses were truly creative and diverse. However, it may be cautiously stated that the common denominator was perceptual or spiritual: "The practice causes the consciousness to be more connected to the body after focusing attention on the body in a way that improves ability to think clearly"; "In my view, during the lesson the brain disengaged from all kinds of thoughts apart from thinking about the movement itself. This gave a sense of contentment and a break from everything, which resulted in renewed energies"; "The very situation of not talking, and performing an isolated, repetitive movement, felt close to meditation. It feels like a better connection with the body, and control of my thoughts".

This theme concluded with a challenging category in which the participants attempted to respond to the question: "Do you think a subconscious process of some kind occurs during the lesson that improves the ability to learn, and if so, what is that process?" About half of the participants did not respond to this question, and of those who did, the vast majority (90\%) said they thought a subconscious process does indeed occur: "By their very nature, the assignments at the Technion require you to constantly use your head, which creates a disconnect with the body, and at some stage leads to 'thinking overload', making it difficult to think clearly. Directing attention to the body, slowing down, and reducing overload on the brain, subconsciously calms the turmoil in your head, and improves ability to learn"; "I think by the very act of 
stopping the day, and the calmness, there's improvement in learning. It's important to take a break, and also to rest, and the lesson provides both, all while exercising and learning".

The participants' responses regarding the subconscious process they experienced during the lesson that resulted in improved learning, were varied, making it difficult to find commonalities. However, it may be cautiously concluded that in the participants' view, the sense of calm typifying Feldenkrais practitioners plays a dominant role in improving the ability to learn.

A summary of the findings obtained in the second theme indicates that most of the participants (about 70\%) felt that doing Feldenkrais exercises improved their ability for theoretical learning after practicing the method. It seems that two main significant factors led to improved concentration and learning: physiological improvement - especially ease and comfort of sitting in a chair, resulting in improved concentration - and mental/emotional improvement - a sense of calm that leads to improved concentration.

\subsection{The Correlation Between the Findings on Research Question 1 and 2}

Examination of this Research Question was conducted in several stages. In the first stage, each of the participants' responses were quantified into one score ranging from 1 to 5: 1=No learning at all; 2=Hardly any learning; $3=$ Partial learning; 4=Some learning; and 5=Significant learning.

In the second stage, each participant received an overall score with reference to Research Question 1 - one overall score for theoretical learning during the lesson - and one overall score with reference to Research Question 2 - theoretical learning in the hours following the lesson.

In the third stage, the correlation between the data obtained from each participant with reference to Research Question 1, and the data pertaining to Research Question 2, was analyzed using SPSS software, in which "learning-during-lesson" described theoretical learning during the Feldenkrais lesson, and "learning-after-lesson" described theoretical learning in the hours following the Feldenkrais lesson.

Descriptive Statistics

Cronbach's alpha reliability test to examine level of theoretical learning during a Feldenkrais lesson, and level of theoretical learning in the hours following a Feldenkrais lesson:

Table 2. Reliability Statistics

Cronbach's alpha No. Items

.770

2

Table 3. Descriptive Statistics

\begin{tabular}{lccccc}
\hline & $\mathrm{N}$ & Minimum & Maximum & Mean & Std. Deviation \\
\hline Learning-after-lesson & 120 & 1.00 & 5.00 & 3.0500 & 1.76735 \\
Learning-during-lesson & 120 & 1.00 & 5.00 & 3.2250 & 1.45788 \\
Valid N (listwise) & 120 & & & & \\
\hline
\end{tabular}

Table 4. Learning-during-lesson

\begin{tabular}{cccccc}
\hline & & Frequency & Percent & Valid Percent & $\begin{array}{c}\text { Cumulative } \\
\text { Percent }\end{array}$ \\
\hline \multirow{6}{*}{ Valid } & 1.00 & 19 & 15.8 & 15.8 & 15.8 \\
& 2.00 & 25 & 20.8 & 20.8 & 36.7 \\
& 3.00 & 20 & 16.7 & 16.7 & 53.3 \\
& 4.00 & 22 & 18.3 & 18.3 & 71.7 \\
& 5.00 & 34 & 28.3 & 28.3 & 100.0 \\
& Total & 120 & 100.0 & 100.0 & \\
\hline
\end{tabular}




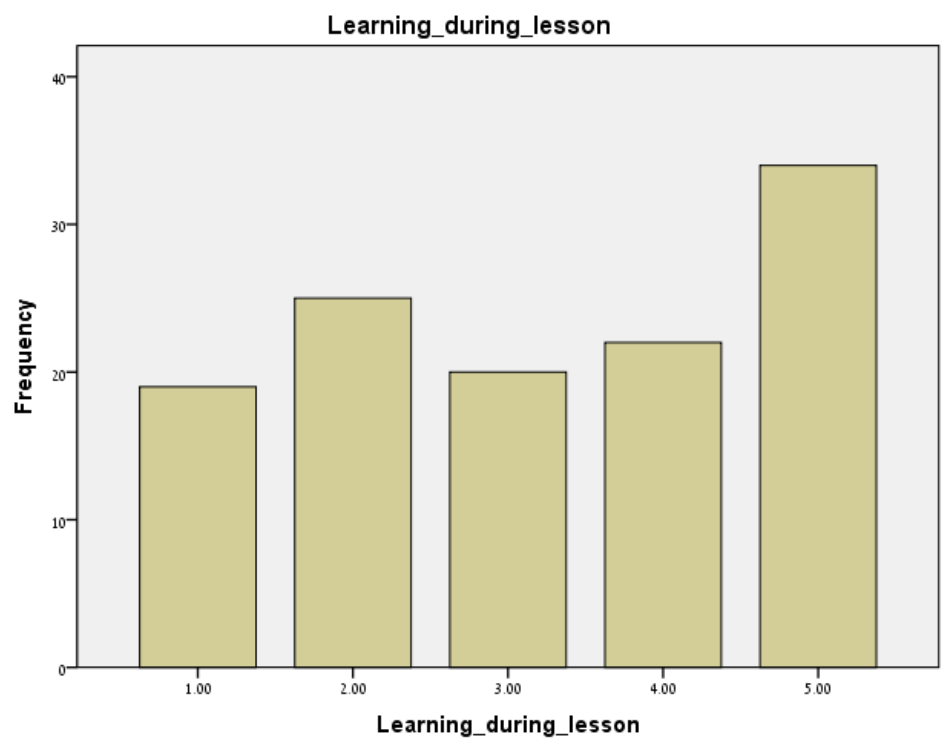

Figure 2. Ability for Theoretical Learning During the Lesson

Table 5. Learning-after-lesson

\begin{tabular}{cccccc}
\hline & & Frequency & Percent & Valid Percent & $\begin{array}{c}\text { Cumulative } \\
\text { Percent }\end{array}$ \\
\hline \multirow{6}{*}{ Valid } & 1.00 & 40 & 33.3 & 33.3 & 33.3 \\
& 2.00 & 17 & 14.2 & 14.2 & 47.5 \\
& 3.00 & 6 & 5.0 & 5.0 & 52.5 \\
& 4.00 & 11 & 9.2 & 9.2 & 61.7 \\
& 5.00 & 46 & 38.3 & 38.3 & 100.0 \\
& Total & 120 & 100.0 & 100.0 & \\
\hline
\end{tabular}

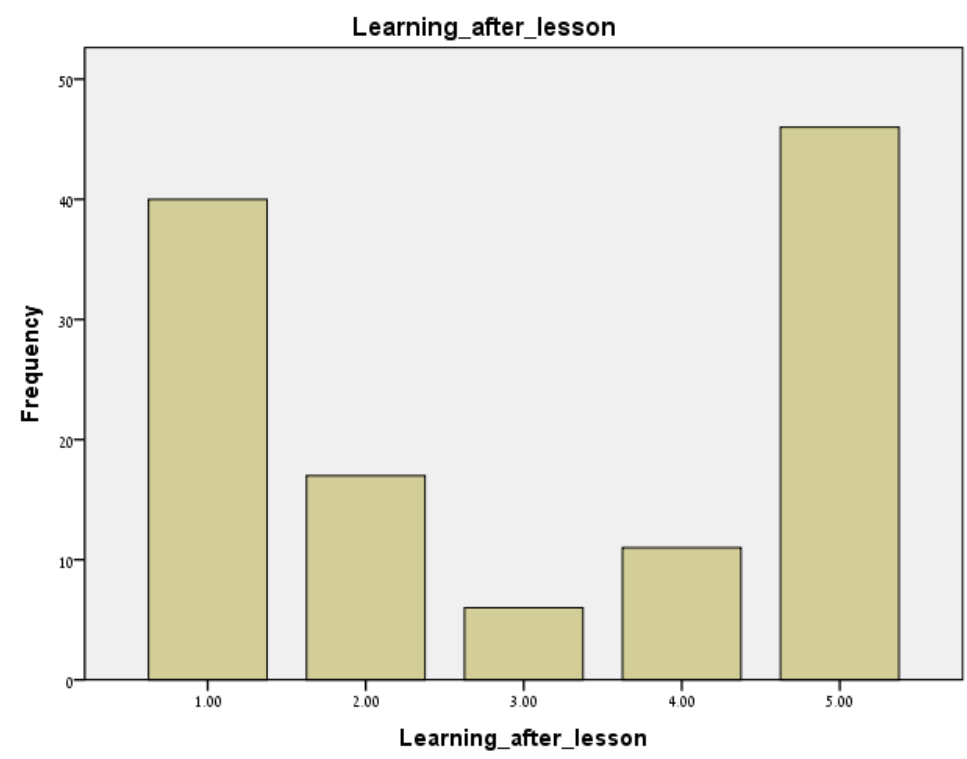

Figure 3. Ability for Theoretical Learning After the Lesson 


\section{$\underline{\text { Statistical Inference }}$}

Spearman's rank correlation test to examine the correlation between theoretical learning during a Feldenkrais lesson, and theoretical learning in the hours following a Feldenkrais lesson:

Table 6. Correlations

\begin{tabular}{|c|c|c|c|}
\hline & & Learning-after-lesson & Learning-during-lesson \\
\hline \multirow{4}{*}{ Learning-after-lesson } & Correlation Coefficient & 1.000 & $.505^{* *}$ \\
\hline & Sig. (2-tailed) & . & .000 \\
\hline & $\mathrm{N}$ & 120 & 120 \\
\hline & Correlation Coefficient & $.505^{* *}$ & 1.000 \\
\hline \multirow[t]{2}{*}{ Learning-during-lesson } & Sig. (2-tailed) & .000 & . \\
\hline & $\mathrm{N}$ & 120 & 120 \\
\hline
\end{tabular}

As we can see, the correlation coefficient (Rho), ranging from 1 to -1 between level of theoretical learning during a Feldenkrais lesson, and theoretical learning in the hours following a Feldenkrais lesson, is 0.505 , indicating a strong positive correlation between them.

Additionally, the sig. (p) value is less than 0.01 , indicating that the correlation is significant $(\mathrm{p}=0.000, \mathrm{r}=0.505)$.

Consequently, $\mathrm{H}_{0}$ can be rejected, and $\mathrm{H}_{1}$ accepted. In other words, there is a (strong) correlation between level of theoretical learning during a Feldenkrais lesson, and theoretical learning in the hours following a Feldenkrais lesson.

$\left(\mathrm{H}_{1}=\right.$ correlation between level of theoretical learning during a Feldenkrais lesson, and theoretical learning in the hours following a Feldenkrais lesson; $\mathrm{H}_{0}=$ no correlation between level of theoretical learning during a Feldenkrais lesson, and theoretical learning in the hours following a Feldenkrais lesson).

Additionally, the sig. (p) value in the quantitative analysis is less than 0.01 , indicating the validity of the study. In other words, the measuring tool examines the research question appropriately.

\section{Discussion and Conclusions}

\subsection{Assimilation of Theoretical Content Taught in the Lessons}

The qualitative findings with reference to Research Question 1 indicated the participants' good ability for theoretical learning during Feldenkrais lessons. These findings stem from movements, sensations, and elements that contribute to any kind of learning, that the participants experienced during the lessons.

First, the movements during the lessons were easy, simple, enjoyable, and similar to movements the participants (and anyone practicing the method) performed in their infancy and childhood. Second, the teacher, an expert in the field, knew how to guide the participants to awaken the "inner guide" within them, and to use movements best suited to them, and which are as close as possible to "natural" movement and learning, to move their body (Feldenkrais, 1967). And third, the participants experienced unconventional movements - rolling the pelvis forward, backward, and to the sides - that encourage the release of physical rigidity in specific areas of the body (lower back and back of the neck), and allow other areas that are unaccustomed to moving (chest) to participate in the movement. Beneficial changes in movements and the body's usual tensions facilitate an easier and more comfortable platform for learning.

Additionally, the participants experienced various positive sensations that may have helped them to be more available for theoretical learning. The participants in the present study, as well as participants in other studies engaging with the Feldenkrais method (Gil, 2013; Hillier \& Worley, 2015), reported a sense of tranquility and calm. Alleviation of pain, which is one of the first aims of Feldenkrais participants (Buchanan, Nelsen, \& Geletta, 2014; Chen, 2017; Morgan, 2017), is a frequently reported sensation as well. Another positive, satisfying sensation the participants experienced is the legitimacy granted by the teacher during the lessons to accept their body and movements as they are. The participants were guided to only move in accordance with what is suited, comfortable, and possible for them; any movement is considered legitimate and good. The participants' sense of tranquility and inner calm, self-acceptance, and acceptance by their environment, facilitate better learning and receptiveness to the environment, including learning theoretical material associated with the lessons.

During the lessons, the participants refined and improved elements that help in any kind of learning, including theoretical learning. For instance, they were guided to pay attention to particular details by means of numerous questions such as: Which limb moves first in the exercise, leading the rest of the body? thus improving their ability to distinguish nuances. 
The unique exercises enabled the participants to improve their ability to adapt, maneuver, and to be resourceful in finding diverse independent solutions in new situations (Alon, 1994). The participants experienced an awakening of their motivation for change and learning in aspects of body, health, emotions, as well as for theoretical learning.

\subsection{Improved Ability to Learn After Doing Feldenkrais Exercises}

The qualitative findings with reference to Research Question 2 indicated the participants' improved ability for theoretical learning in the hours following the lesson. These changes can be explained by means of two positive processes that occur when practicing the method. One is a mental process of tranquility and improved concentration, and the other is a physiological process of pain alleviation, and the ability to sit and learn for longer without pain. As described in the literature on concentration difficulties (Crispel, 2017; Hen, 2018; Lufi, Elner, \& Levi, 2004), and on the difficulties of sitting in a chair (Vergara \& Page, 2002; Womersley \& May, 2006), both disrupt learning. Consequently, improvement in these aspects during participation in Feldenkrais lessons will lead naturally and immediately to improved ability to learn.

\subsection{Correlation Between Ability to Learn During and After the Lessons}

The quantitative findings indicated a positive, even significant, correlation between the findings pertaining to Research Question 1, and the findings pertaining to Research Question 2. In other words, the participants who experienced high ability for theoretical learning during the lesson, also experienced high ability for theoretical learning in the hours following the lesson.

The positive process that took place during the lessons, and facilitated improvement in learning, is primarily associated with the nervous system. Over the years, the participants' (as all people's) nervous system has become accustomed to and "fixed" on transmitting particular neural signals from the brain to particular muscles to perform particular actions. For all the reasons stated in the findings pertaining to Research Question 1 (gentle movements, finding the inner guide, and so forth), and based on the research (Gil, 2013; Morgan, 2017; McIvor, 2015; Teixeira-Machado et al., 2015), doing Feldenkrais exercises facilitates changes in conductivity and pathways between the brain and the muscles. For instance, practicing the method enables muscles that usually receive multiple neural signals to contract, e.g., the neck and lumbar muscles, an opportunity to move without muscular strain, but rather by moving the joints in the area, and regenerated movement of adjacent areas (chest) that are usually fixed (Gil, 2013).

In addition to creating these new pathways, which stimulate blocked pathways into action, remove tension from wellworn pathways, new synaptic connections are formed, and new neural signals are transmitted. This awakening of the nervous system created in the participants additional forms and coordination in all the neural pathways, and beneficial changes in the pathways between the back and the rest of the body, and between the brain and all the other parts of the body (Alon, 1995). It may be assumed that it is these better pathways between the back and the rest of the body that made it easier for the participants to sit for longer after the lessons, and the pathways between the brain and the rest of the body that created the tranquility, calm, and balance the participants felt, all of which improved their ability to learn.

\subsection{Uniqueness of the Present Study}

The study examined, for the first time, the correlation between participating in Feldenkrais lessons and improvement in the participants' ability to learn. The results indicate improvement in the participants' ability to learn, both during and after the lessons.

Dr. Moshé Feldenkrais described and explained human ability to learn, and how movement in accordance with his method can promote this ability. The present study scientifically proves, for the first time, the veracity of his writings and descriptions concerning human ability to learn.

\subsection{Limitations of the Study}

All the lessons were analyzed as a whole, and attention was not given during data analysis to possible differences between the participants' responses at the beginning and at the end of the semester.

The questionnaires were not identical for all the lessons, and it seems that the responses were influenced by their quality. The question asked at the end of the lesson on the relationship between the head, neck, and shoulders, was: "In your view, did learning occur during the lesson? If so, what was it?" It seems that the wording of this question was too broad, making it difficult for some participants to focus their response. On the other hand, the instruction given at the end of the lesson on differences between upper and lower limbs, was: "Even though I didn't give any explanation on the Feldenkrais method during the lesson, write down what you learned about it". This question yielded richer responses, and possibly contributed to their focus, and also to their categorization as more positive in the data analysis.

The "truth" presented in the study is correct and relevant for the time it was conducted. Older participants, or participants with lower intellectual abilities, will possibly respond differently. 


\subsection{Summary and Research Recommendations}

It seems that doing Feldenkrais exercises not only yields mental/emotional benefits such as improved mood, and heightened awareness of the body, as proved in other studies (Gil, 2013; Netz \& Lidor, 2003; Teixeira-Machado et al., 2015), or only physiological benefits such as alleviation of pain, as claimed in previous studies (Chen, 2017). The present study reinforces, even proves, the veracity of viewing the Feldenkrais method as a method that stimulates and regenerates the brain (McIvor, 2015; Morgan, 2017).

It may be cautiously proposed that Feldenkrais exercises can help participants to "unblock" stressful neural pathways, encourage activation of new neural pathways, alleviate various aches and pains (especially back pain when sitting in a chair), reduce mental stress, and create recognition, confidence, and awareness of how we can improve and refine ourselves - all of which led, in the present study, to improved concentration, and, in turn, to improved ability to learn after the lessons.

In further research on this subject, developing a uniform questionnaire for all the lessons is recommended, and comparing the participants' responses at the beginning and end of the study. Additional research possibilities include comparing between different participant populations, whether according to gender or ethnic origin.

\section{References}

Alon, R. (1994). Mindful spontaneity: Returning to natural movement. Berkeley, CA: North Atlantic Books.

Bitter, F., Hillier, S., \& Civetta, L. (2011). Change in dexterity with sensory awareness training: A randomised controlled trial. Perceptual and Motor Skills, 112(3), 783-798. https://doi.org/10.2466/15.22.PMS.112.3.783-798

Buchanan, P. A., Nelsen, N. L., \& Geletta, S. (2014). A survey of characteristics and practice patterns. BMC Complementary and Alternative Medicine, 14, 217. https://doi.org/10.1186/1472-6882-14-217

Chen, L., \& Michalsen, A. (2017). Management of chronic pain using complementary and integrative medicine. BMJ: British Medical Journal, 357. https://doi.org/10.1136/bmj.j1284

Chinn, J., Trujilo, D., Kegerreis, S., \& Worrel, T. (1994). Effect of a Feldenkrais intervention on symptomatic subjects performing a functional reach. Isokinetics and Exercise Science, 4(4), 131-136. https://doi.org/10.3233/IES-19944401

Cohen-Gil, M. (2013). The Israelis who wished to cure the world. Jerusalem: Keter.

Creswell, J. W. (2009). Research design (3rd ed.) Lincoln, NE: University of Nebraska Press.

Crispel, O. (2017). Acquiring knowledge regarding learning disabilities and the significance of "caring" in teacher education. Studies in Education, 15-16, 417-438. University of Haifa.

Feldenkrais, M. (1967). Practical ability improvement. Tel Aviv: The Feldenkrais Institute.

Feldenkrais, M. (1976). Chapters in my method. Tel Aviv: Aleph.

Feldenkrais, M. (1985). The potent self: A guide to spontaneity. New York, NY: Harper \& Row.

Feldenkrais, M. (1993). Body awareness as healing therapy: The case of Nora. Berkeley, CA: Frog Books.

Gil, I. (2013). A new educational program for improving posture and quality of life among students by using the Feldenkrais and MAP methods. Doctoral dissertation, Ohalo Academic College of Education, Israel.

Hen, M. (2018). Academic procrastination and feelings toward procrastination in LD and non-LD students: Preliminary insights for future intervention. Journal of Prevention \& Intervention in the Community, 46(2), 199-212. https://doi.org/10.1080/10852352.2016.1198173

Hillier, S., \& Worley, A. (2015). The effectiveness of the Feldenkrais method: A systematic review of the evidence. Evidence-Based Complementary and Alternative Medicine, 2015(1), 1-12. https://doi.org/doi:10.1155/2015/752160

Hillier, S., Porter, L., Jackson, K., \& Petkov, J. (2010). The effects of Feldenkrais classes on the health and function of an ageing Australian sample: A pilot study. The Open Rehabilitation Journal, 3(1), 62-66. https://doi.org/10.2174/1874943701003010062

Kegerreis, S., \& Brown, E. (1991). Electromyographic activity of trunk musculature during a Feldenkrais awareness through movement lesson. Isokinetics and Exercise Science, 1(4), 216-221. https://doi.org/10.3233/IES-1991-1407

Laumer, U., Bauer, M., Fichter, M., \& Helmut, M. (1997). Therapeutische Effekte der Feldenkrais-Methode 'Bewußtheit durch Bewegung' bei Patienten mit Eßstörungen [Therapeutic effects of the Feldenkrais method 'awareness through movement' in patients with eating disorders]. Psychotherapie Psychosomatik Medizinische Psychologie, 47(5), 170180. 
Löwe, B., Breining, K., Wilke, S., Wellmann, R., Zipfel, S., \& Eich, W. (2002). Quantitative and qualitative effects of Feldenkrais, progressive muscle relaxation, and standard medical treatment in patients after acute myocardial infarction. Psychotherapy Research, 12(2), 179-191. https://doi.org/10.1093/ptr/12.2.179

Lufi, D., Elner, I., \& Levi, N. (2004). Assessment of intellectual ability, cognitive functioning, and personality characteristics of adolescents with learning disabilities. Issues in Special Education \& Rehabilitation, 19(1), 69-82. https://www.jstor.org/stable/i23453155

McIvor, A. (2015). Book review: A compendium of essays on alternative therapy. Perspectives in Public Health, 135(2), 108.

Morgan, J. (2017). No miracle, just our brain. The Lancet Neurology, 16(1), 32. https://doi.org/10.1016/s14744422(16)30089-8

Nambi, G., Trivedi, P., Momin, S., Patel, S., \& Pancholi, D. (2014). Comparative effect of Pilates and Feldenkrais intervention on functional balance and quality of life in ambulatory geriatric population: A randomized controlled study. International Journal of Health Sciences and Research, 4(3), 71-77.

Netz, Y., \& Lidor, R. (2003). Mood alterations in mindful versus aerobic exercise modes. The Journal of Psychology, 137(5), 405-419. https://doi.org/10.1080/00223980309600624

Ruth, S., \& Kegerreis, S. (1992). Facilitating cervical flexion using a Feldenkrais method: Awareness through movement. Journal of Orthopaedic and Sports Physical Therapy, 16(1), 25-29. https://www.jospt.org/doi/10.2519/jospt.1992.16.1.25

Smith, A. L., Kolt, G. S., \& McConville, J. C. (2001). The effect of the Feldenkrais method on pain and anxiety in people experiencing chronic low back pain. New Zealand Journal of Physiotherapy, 29(1), 6-14.

Stephens, J., Davidson, J., DeRosa, J., Kriz, M., \& Saltzman, N. (2006). Lengthening the hamstring muscles without stretching using "awareness through movement". Physical Therapy, 86(12), 1641-1650. https://doi.org/10.2522/ptj.20040208

Stephens, J., DuShuttle, D., Hatcher, C., Shmunes, J., \& Slaninka, C. (2001). Use of awareness through movement improves balance and balance confidence in people with multiple sclerosis: A randomized controlled study. Neurology Report, 25(2), 39-49. https://doi.org/10.1097/01253086-200125020-00002

Teixeira-Machado, L., Araújo, F. M., Cunha, F. A., Menezes, M., Menezes, T., \& Melo DeSantana, J. (2015). Feldenkrais method-based exercise improves quality of life in individuals with Parkinson's disease: A controlled, randomized clinical trial. Alternative Therapies in Health and Medicine, 21(1), 8-14. https://doi.org/10.1016/j.jpain.2015.01.471

Ullmann, G., Williams, H. G., Hussey, J., Durstine, J. L., \& McClenaghan, B. A. (2010). Effects of Feldenkrais exercises on balance, mobility, balance confidence, and gait performance in community-dwelling adults age 65 and older. Journal of Alternative and Complementary Medicine, 16(1), 97-105. https://doi.org/10.1089/acm.2008.0612

Vergara, M., \& Page, A. (2002). Relationship between comfort and back posture and mobility in sitting-posture. Applied Ergonomics, 33(1), 1-8. https://doi.org/10.1016/S0003-6870(01)00056-4

Vrantsidis, F., Hill, K. D., Moore, K., Webb, R., Hunt, S., \& Dowson, L. (2009). Getting grounded gracefully: Effectiveness and acceptability of Feldenkrais in improving balance. Journal of Aging and Physical Activity, 17(1), 57-76. https://doi.org/10.1123/japa.17.1.57

Womersley, L., \& May, S. (2006). Sitting posture of subjects with postural backache. Journal of Manipulative and Physiological Therapeutics, 29(3), 213-218. https://doi.org/10.1016/j.jmpt.2006.01.002

\section{Copyrights}

Copyright for this article is retained by the author(s), with first publication rights granted to the journal.

This is an open-access article distributed under the terms and conditions of the Creative Commons Attribution license which permits unrestricted use, distribution, and reproduction in any medium, provided the original work is properly cited. 